\title{
Thermodynamique des pompes et turbines hydrauliques
}

\section{Thermodymamics of hydraulic pumps and turbimes}

\author{
Robert Legendre \\ Membre de l'Académie des Sciences \\ Haut Conseiller Scientifique à l'O.N.E.R.A.
}

\section{Introduction}

La faible compressibilité des liquides est partiellement compensée par leur forte densité. C'est pourquoi, par exemple, la célérité du son dans l'eau est moins de cinq fois plus grande que dans l'air. Il est donc parfaitement légitime d'appliquer aux pompes et aux turbines hydrauliques les lois de la thermodynamique valables pour toutes les turbomachines à fluides compressibles, indépendemment du fluide véhiculé.

Toutefois, les considérations suivantes ne sont illustrées que pour l'eau douce, non émulsionnée par des gaz modifiant sensiblement la compressibilité. En outre, la pression doit être supérieure à la tension de vapeur, majorée de la tension des gaz dissous, au moins dans les états de référence.

Non seulement l'eau est peu compressible mais elle est pratiquement indilatable et son entropie ne dépend guère que de la température. En conséquence, une détente isentropique est sensiblement isotherme et il est effectivement justifié d'établir l'essentiel des propriétés des machines en supposant l'eau indilatable et incompressible.

Par contre, les pertes par turbulence et frottement, et en définitive par viscosité, dépendent de l'énergie dégradée en chaleur qui provoque de légers accroissements d'entropie et de température. Il est aujourd'hui possible d'effectuer des mesures de température de très haute précision, permettant d'accéder à une connaissance des pertes, indépendemment de la mesure du rendement mécanique.

L'énoncé du principe de la détermination du rendement isentropique remonte à 1920 [1]. La méthode de mesure convient particulièrement bien aux turbines et pompes des hautes chutes d'eau, véhiculant de l'eau froide [3,4] mais son emploi fut étendu aux pompes véhiculant de l'eau chaude [2] ou mème du pétrole [5]. De telles généralisations ne sont pas discutées ici, car il est inutile de répéter les précautions à prendre pour compenser les effets d'une imparfaite adiabaticité et des irrégularités de température dans les sections d'entrée et de sortie des machines.

L'objectif fixé est l'élimination de l'emploi des valeurs incertaines des chaleurs spécifiques aux pressions élevées, grâce à la construction méthodique d'un potentiel thermodynamique.

\section{Thermodynamique de l'eau}

Toutes les propriétés thermodynamiques de l'eau sont rassemblées dans le potentiel à pression constante de Gibbs $G(T, p)$, où $T$ est la température et $p$ la pression. Ce potentiel dépend assez peu de la pression pour être bien représenté par un développement en série limitée en fonction de $p$, ou plutôt en fonction de $p-p_{0}$, où $p_{0}$ est la pression atmosphérique, afin que les coef ficients aient une signification simple à cette pression de référence, fixée conventionnellement à 1 bar ou $10^{5}$ pascals.

Le volume massique $v$ et le coefficient de compressibilité $\beta$ sont déduits d'un développement de $G$ si convergent qu'une représentation satisfaisante est obtenue, même si $p$ s'éléve à plusieurs dizaines d'atmosphères, lorsque trois ou quatre termes sont retenus.

$$
\begin{aligned}
G(T, p)= & G_{0}(T)+G_{1}(T)\left(p-p_{0}\right) \\
+ & G_{2}(T)\left(p-p_{0}\right)^{2}+G_{3}(T)\left(p-p_{0}\right)^{3}+\ldots \\
v= & \partial G / \partial p=G_{1}+ \\
& \quad+2 G_{2}\left(p-p_{0}\right)+3 G_{3}\left(p-p_{0}\right)^{2}+\ldots \\
\beta= & \partial \ln v / \partial p= \\
& \quad-2 G_{2}-6 G_{3}\left(p-p_{0}\right)-\ldots \\
& \quad+2 G_{2}\left(p-p_{0}\right)+3 G_{3}\left(p-p_{0}\right)^{2}+\ldots \\
\sim & -2 G_{2} / G_{1}-\left(6 G_{3} / G_{1}-4 G_{2}^{2} / G_{1}^{2}\right)\left(p-p_{0}\right)
\end{aligned}
$$


L'entropie $S$, l'enthalpie $H$, la chaleur spécifique $C_{p}$ sont :

$$
\begin{aligned}
& S=-\partial G / \partial T=-G_{0}^{\prime}-G_{1}^{\prime}\left(p-p_{0}\right)-G_{2}^{\prime}\left(p-p_{0}\right)^{2}-\ldots \\
& \begin{aligned}
\mathrm{H}=\mathrm{C}^{\mathrm{te}}+G+T S= \\
\quad=H_{0}+H_{1}\left(p-p_{0}\right)+H_{2}\left(p-p_{0}\right)^{2}+\ldots \\
=\mathrm{C}^{\mathrm{te}}+G_{0}-T G_{0}^{\prime}+\left(G_{1}-T G_{1}^{\prime}\right)\left(p-p_{0}\right)+\ldots \\
C_{p}=\partial H / \partial T=-T G_{0}^{\prime \prime}-T G_{1}^{\prime \prime}\left(p-p_{0}\right)-\ldots
\end{aligned}
\end{aligned}
$$

C'est en fait $H$ et non $G$ qui est accessible à la mesure calorimétrique directe. Comme les fonctions $G_{1}, G_{2} \ldots$ figurent dans l'équation d'état $v(T, p)$, il suffit de déduire $G_{0}$ de $H_{0}$ par :

$$
G_{0}=-\int_{0}^{T}\left(T / T^{\prime}-1\right) d H_{0}\left(T^{\prime}\right)
$$

Toutes les propriétés thermodynamiques sont ainsi déduites de l'équation d'état et de mesures calorimétriques à la pression atmosphérique.

En principe, $G_{0}$ est intégré à partir du zéro absolu de température et tient compte de la chaleur de fusion de la glace, mais il importe peu ici que $S$ et $H$ soient définis à des constantes près. Leurs origines peuvent être prises, par exemple, à $0^{\circ} \mathrm{C}$. Alors $G$ est modifié, sans inconvénient, par une fonction linéaire de la température.

\section{Discussion des ordres de grandeur}

Le volume massique à la pression atmosphérique $v_{1}(T)=G_{1}(T)$ présente, pour l'eau, un minimum à $4^{\circ} \mathrm{C}$ et varie très peu au voisinage de ce minimum dans le domaine retenant ici l'intérêt. L'eau est donc effectivement indilatable, $v_{1}$ restant constamment au voisinage de $10^{-3} \mathrm{~m}^{3} / \mathrm{kg}$.

Le coefficient de compressibilité $\beta$ à la pression atmosphérique : $-2 G_{2} / G_{1}$ est très faible. Il varie quelque peu avec la température :

$\begin{array}{llll}\text { température } & 0^{\circ} \mathrm{C} & 20^{\circ} \mathrm{C} & 60^{\circ} \mathrm{C} \\ \beta \times 10^{5} & 5,0 & 4,6 & 4,5\end{array}$

où $\beta$ est exprimé en atmosphères à la puissance moins un. Il faut encore le diviser par $10^{5}$ pour l'exprimer en pascals à la puissance moins un. Les applications visées intéressent quelques dizaines d'atmosphères mais, pour l'étude des seules propriétés mécaniques des machines hydrauliques, la compressibilité de l'eau est négligeable.

Le coefficient $\beta$ diminue lorsque la pression s'élève, d'environ $0,1 \%$ par atmosphère à $20^{\circ} \mathrm{C}$ et de manière quelque peu variable avec la température, mais les effets de la pression sur $v$ sont si faibles que le minimum de celui-ci reste au voisinage de $4^{\circ} \mathrm{C}$ lorsque la pression s'élève.

La chaleur spécifique $C_{p}$ est également peu variable avec la température dans le domaine considéré. Elle est voisine de $4,18 \mathrm{~kJ}$ par $\mathrm{kg}$ et par degré, soit de $4,180 \mathrm{~m}^{2} / \mathrm{s}^{2} \mathrm{C}$

$$
d H=C_{p} d T+v d p
$$

le second terme est $100 \mathrm{~m}^{2} / \mathrm{s}^{2}$ pour une variation de pression de une atmosphère ; il est donc plus de quarante fois plus petit que le premier terme pour une variation de température de $1^{\circ} \mathrm{C}$, mais ce sont les variations de pression qui sont utilisées dans les machines hydrauliques.

Pour discuter l'évolution isentropique, il suffit de considérer une petite variation $d S$ au cours de laquelle la variation de température $d T$ est liée à la variation de pression $d p$ par :

$$
\frac{d T}{d p}=-\frac{G_{1}^{\prime}+2 G_{2}^{\prime}\left(p-p_{0}\right)+\ldots}{G_{0}^{\prime \prime}+G_{1}^{\prime \prime}\left(p-p_{0}\right)+\ldots}
$$

Puisque $G_{1}^{\prime}$ est nul à $4^{\circ} \mathrm{C}$ et que le minimum de $v$ reste situé au voisinage de cette température lorsque la pression croit, il est confirmé que l'évolution isentropique est isotherme à $4^{\circ} \mathrm{C}$. Elle reste sensiblement isotherme à toutes les températures ambiantes.

$G_{0}^{\prime \prime}=-C_{p} / T$ est négatif tandis que $G_{1}^{\prime}$ est négatif au-dessous de $4^{\circ} \mathrm{C}$ et positif au-dessus mais la variation isentropique de température est toujours faible.

Les calculs ci-dessus sont suffisants pour une discussion mais, en raison des faibles variations de température à la traversée d'une turbo-machine, il ne faut pas négliger de tenir compte des faibles variations isentropiques.

La célérité du son $a$ est définie par:

$$
1 / a^{2}=-\left(1 / v^{2}\right)(d v / d p)_{S}
$$

Il suffira de l'évaluer à la pression atmosphérique comme le moyen d'accès le plus précis à la fonction $G_{2}(T)$ ou au coefficient de compressibilité $\beta$ :

$$
\begin{aligned}
1 / a^{2} & =-2 G_{2} / G_{1}^{2}+G_{1}^{\prime 2} / G_{1}^{2} G_{0}^{\prime \prime} \\
\beta & =v_{1}\left(1 / a^{2}+T \beta^{\prime 2} / C p\right)
\end{aligned}
$$

où $\beta^{\prime}=-\partial \ln v / \partial T$ est le coefficient de dilatabilité à pression constante qui est très faible.

\section{Rendement isentropique}

L'établissement du diagramme de Mollier $(S, H)$ pour l'eau à l'état liquide, aux températures ambiantes

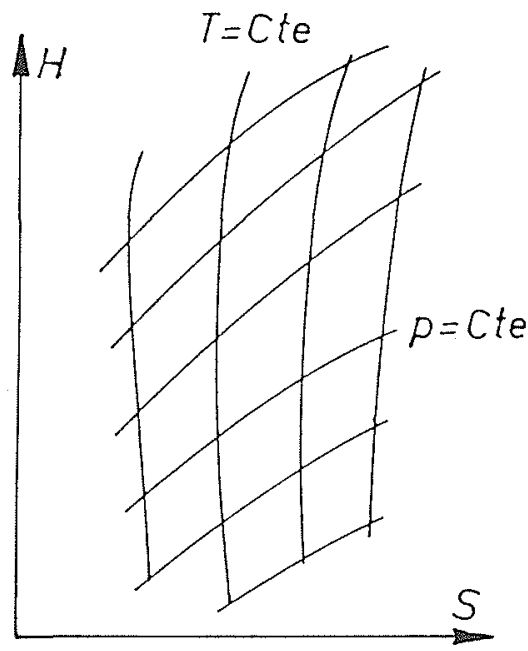

Figure 1 
usuelles, dispense de l'approximation acceptée par la limitation de la série représentant $G(T, p)$

Par un choix convenable des échelles (fig. 1), il est commode d'obtenir que la pente des isobares

$$
d H / d S=T
$$

soit de l'ordre de 1 dans le domaine limité utilisé ; les isobares sont approximativement des droites parallèles inclinées à $45^{\circ}$ sur les axes.

Les isothermes s'écartent peu des isentropes. Toutefois, en raison de la forte dilatation d'échelle pour l'entropie, d'une part, des exigences de précision qui apparaîtront plus loin, d'autre part, il est utile de tenir compte de la pente et de la courbure des isothermes.

L'évolution de l'état d'un fluide entre l'aspiration et le refoulement d'une turbopompe est représentée par les couples de points $A, A_{0}$ et $B, B_{0}$ (fig. 2)

Le point $A$ est à l'intersection de l'isotherme $T_{A}$ et de l'isobare $p_{A}$ de la pression au centre de la section d'entrée. Il lui correspondent l'entropie $S_{A}$ et l'enthalpie $H_{A}$.

Le point $A_{0}$ représente l'état d'arrêt isentropique à partir de $A$. Il est sur l'isentrope $S_{A}$ à la cote

$$
H_{A_{O}}=H_{A}+V_{A}^{2} / 2+g z_{A}
$$

où

$-V_{A}$ est la vitesse débitante dans la section de mesure à l'entrée,

- $g$ l'accélération de la pesanteur

$-z_{A}$ la cote du centre de la section d'entrée au-dessus d'une horizontale de référence de la machine, telle que l'axe d'un arbre horizontal.

Les points $B, B_{0}$ sont définis comme $A, A_{0}$ mais pour les grandeurs mesurées dans la section de sortie.

Les points $C, C_{0}$ sont des références de compression isentropique à partir de $A, A_{0}$. Ils sont sur l'isentrope $S_{A}$. Le point $C$ est sur l'isobare $p_{B}$, à l'enthalpie $H_{C}$ et la cote d'enthalpie de $C_{0}$ est $H_{C_{0}}=H_{C}+g z_{B}$

Le travail dépensé sur l'arbre de la pompe pour une évolution adiabatique est $H_{B}-H_{A_{0}}$ Le travail qui serait dépensé pour une évolution isentropique jusqu'à une vitesse nulle au refoulement est $H_{C_{0}}-H_{A_{0}}$. Le rendement isentropique est :

$$
\eta=\frac{H_{C_{0}}-H_{A_{0}}}{H_{B_{0}}-H_{A_{0}}}
$$

L'évolution de l'eau dans une turbine hydraulique est analogue mais $C, C_{0}$ sont alors au dessous de $A, A_{0}$ (fig. 3) et la définition retenue pour le rendement doit être adaptée :

$$
\eta=\frac{H_{A_{0}}-H_{B_{0}}}{H_{A_{0}}-H_{C_{0}}}
$$

Dans les deux cas, si $\delta p$ est la valeur absolue de la variation de pression lors de la traversée de la machine, éventuellement corrigée des effets de vitesse et de cote $z$, la variation d'entropie est approximativement fournie par :

$$
\delta S \sim(1-\eta) v_{1} \delta p / T_{p}
$$

Comme, par ailleurs,

$$
\delta S \sim C_{p} \delta T / T
$$

où $\delta T$ est l'accroissement de température de l'eau à la traversée de la machine :

$$
\delta T \sim(1-\eta) v_{1} \delta p / C_{p}
$$

Pour une turbine Pelton fonctionnant sous une différence de pression de 80 atmosphères, $v_{1} \delta p$ est de l'ordre de $8000 \mathrm{~m}^{2} / \mathrm{s}^{2}$ et $v_{1} \delta p / C_{p}$ est de l'ordre de $2^{\circ} \mathrm{C}$. Il faut donc mesurer $T$ avec une précision d'une petite fraction de degré pour accéder à une évaluation significative de $\eta$.

\section{Conclusions}

Bien que les isothermes soient voisines des isentropes sur le diagramme de Mollier de l'eau aux températures ambiantes, il est utile de tenir compte des légers écarts

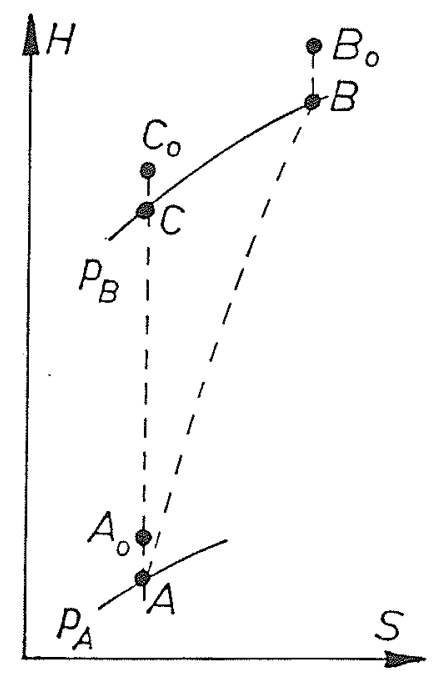

Figure 2

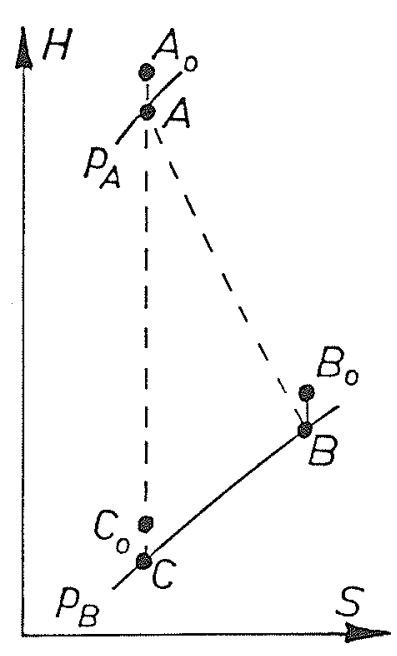

Figure 3 
avec une bonne précision en raison des faibles variations de température de l'eau à la traversée d'une machine hydraulique, même lorsque la variation de pression est importante.

Le résultat est obtenu si, aux chaleurs spécifiques à haute pression relevées dans la documentation, sans souci de cohérence avec la loi d'état, sont substituées celles qui dérivent đu potentiel thermodynamique à pression constante rassemblant, sans risque de contradiction, toutes les propriétés mécaniques et thermiques de l'eau.

\section{Références}

BARBILLION L., POIRSON A. - Sur une méthode thermometrique de mesure du rendement des turbines hydrauliques. La Houille Blanche, 1920, pp. 217.

CHALEIL J. - Détermination du rendement des pompes alimentaires, par la méthode thermodynamique. L'Eau, Septembre 1966, pp. 1 .

GABAUDAN J., THOMAS P.M., GALIAN J. - Evolution de la méthode thermodynamique de la détermination du rendement des machines hydrauliques. La Houille Blanche, 1966-4, pp. 433 .
THOM A.S., FOORD T.R., PEARSON W.K. - Thermodynamic measurement of Pelton turbine efficiency. Water Power \& Dam Construction, Feb. 1975, pp. 64.

WITT K. - Thermodynamisches Messen in der Olhydraulik. Olhydraulik und Pneumatik 20 - 1976 - 6, pp. 416.

LEGENDRE R. - Thermodynamique des fluides. Note Technique ONERA, 1982-1. 